\title{
Relations génétiques entre populations de taurins ou zébus d'Afrique de l'Ouest et taurins européens
}

\author{
Rémy Queval ${ }^{a}$, Katayoun Moazami-Goudarzi ${ }^{\mathrm{b} *}$, Denis Laloë ${ }^{\mathrm{c}}$, \\ Jean-Claude Mériaux ${ }^{\mathrm{d}}$, François Grosclaude ${ }^{\mathrm{b}}$
}

a Centre international de recherche-développement sur l'élevage en zone subhumide, BP 454 Bobo Dioulasso, Burkina-Faso

b Laboratoire de génétique biochimique et de cytogénétique, Institut national de la recherche agronomique, 78352 Jouy-en-Josas cedex, France

c Station de génétique quantitative et appliquée, Institut national de la recherche agronomique, 78352 Jouy-en-Josas cedex, France

d Laboratoire d'analyses génétiques pour les espèces animales, 78352 Jouy-en-Josas cedex, France

(Reçu le 6 avril 1998; accepté le 15 juin 1998)

\begin{abstract}
Genetic relationships among West-African taurine or indicine populations and European cattle breeds. The polymorphism of 16 genetic systems, the 11 known blood group systems in cattle $\left(A, B, C, F, J, L, M, S, Z, R^{\prime}, T^{\prime}\right)$ and five blood protein loci (serum albumin and transferrin, erythrocyte haemoglobin, purine nucleoside phosphorylase and phosphoglucomutase) - has been analysed in ten WestAfrican taurine and indicine populations, and submitted to classification methods and correspondence analysis in an attempt to clarify the genetic relationships between these populations. Since 12 loci are common to this study and an earlier one concerning French breeds, the data of both studies have been pooled and analysed by the same methods. The results show a clear separation between zebus (Bos indicus) and taurines (Bos taurus), as well as between African and European taurines. The correspondence analysis highlights the discriminant effect of alleles specific to Zebu $\left(T f^{F}, A l^{S}\right)$ or more frequent in Zebu than in taurines $\left(H b^{B}\right)$, and that of alleles mainly found in African cattle $\left(F^{-}, C^{-}, C^{C_{1-}}\right)$. On the other hand, the methods used could not clearly separate the West-African taurine populations or the European cattle breeds. (C) Inra/Elsevier, Paris

biochemical polymorphism / phylogenetic relationship / taurines / zebus / WestAfrica
\end{abstract}

* Correspondance et tirés à part

Courriel : goudarzi@biotec.jouy.inra.fr 
Résumé - Le polymorphisme de 16 systèmes génétiques, les 11 systèmes de groupes sanguins connus chez les bovins $\left(A, B, C, F, J, L, M, S, Z, R^{\prime}, T^{\prime}\right)$ et cinq locus de protéines sanguines (albumine et transferrine sériques, hémoglobine, purine nucléoside phosphorylase et phosphoglucomutase érythrocytaires), a été analysé dans dix populations de taurins ou de zébus de l'Afrique de l'Ouest et traité par des méthodes de classification et par analyse factorielle des correspondances pour tenter de préciser les relations de parenté existant entre ces populations. Douze systèmes génétiques (groupes sanguins et transferrine) étant communs à cette étude et au travail antérieur sur les races françaises, les données des deux sources ont été regroupées et analysées par les mêmes méthodes. Ces analyses séparent clairement les zébus (Bos indicus) des taurins (Bos taurus), ainsi que les taurins africains des taurins européens. L'analyse factorielle des correspondances fait ressortir le rôle discriminant d'allèles propres aux zébus $\left(T f^{F}, A l^{S}\right)$ ou plus fréquents chez ceux-ci $\left(H b^{B}\right)$, et celui d'allèles paraissant largement spécifiques des bovins africains $\left(F^{-}, C^{-}, C^{C_{1-}}\right)$. En revanche, les outils utilisés ne permettent de séparer d'une manière robuste, ni les populations taurines de l'ensemble Ouest africain, ni les races bovines européennes. (c) Inra/Elsevier, Paris polymorphisme biochimique / relations phylogénétiques / taurins / zébus / Afrique de l'Ouest

\section{INTRODUCTION}

Le bétail d'Afrique de l'ouest se caractérise par une grande diversité, puisqu'il comprend des taurins (Bos taurus), des zébus (Bos indicus), ainsi que de nombreux types intermédiaires résultant de brassages plus ou moins anciens et importants, liés aux migrations humaines et au pastoralisme. Les zébus occupent les régions les plus sèches, comme le Sahel, alors que les taurins se trouvent dans les zones plus humides, infestées par les glossines, ou «mouches tsé-tsé», vectrices principales des trypanosomes [23]. Ces taurins sont en effet trypanotolérants, ce qui est un atout réel pour ces zones, compte tenu de la difficulté de mise au point d'un vaccin et des inconvénients de la lutte par voie chimique.

Selon Epstein [9], les premiers bovins domestiques implantés en Afrique étaient des animaux sans bosse, à cornes longues. D'abord introduits en Egypte, dans la seconde moitié du cinquième millénaire avant J.-C., à partir du foyer de domestication de l'Asie du Sud-Ouest, ces taurins à cornes longues se sont répandus dans tout le tiers nord et nord-est du continent africain. Ils ne sont plus représentés, actuellement, que par les types N'Dama (Afrique de l'ouest) et Kouri (bassin du lac Tchad). Au cours du deuxième millénaire avant J.-C., des taurins à cornes courtes ont suivi la même voie de pénétration, conduisant au groupe de bovins à cornes courtes de l'Afrique de l'Ouest (West African Shorthorn). Ce groupe est représenté par des types disparates, aux appellations diverses (Bakosi, Baoulé, Lagunaire, Muturu, Nuba, Somba) et comporte aussi des variantes régionales. Les zébus représentent un troisième apport, plus tardif. Il s'agissait d'animaux à bosse thoracique, dont l'introduction en Afrique semble avoir commencé vers le quatrième siècle après J.-C., et dont la diffusion s'est intensifiée après l'invasion arabe (669 après J.-C.). Ces divers types génétiques se sont largement mélangés dans certaines zones. Selon Epstein [9], tous les bovins africains proviendraient d'une même région d'origine, l'Asie du SudOuest. Les taurins à cornes longues y auraient d'abord été domestiqués, au 
début du cinquième millénaire avant J.-C., sans doute en Mésopotamie, à partir de la population locale de bœufs sauvages. C'est ensuite à partir de ces taurins à cornes longues qu'auraient été sélectionnés les taurins à cornes courtes, probablement en Elam, au cours du quatrième millénaire avant J.-C., ainsi que les zébus, à partir de la même période, peut-être dans la steppe semiaride bordant l'Est du grand désert salé d'Iran. Toutefois les travaux de Loftus et al. [24] sur l'ADN mitochondrial des bovins tendent à remettre en cause la thèse d'Epstein sur l'origine des zébus. En effet, l'analyse des différences de séquence observées entre l'ADN mitochondrial des taurins et celui des zébus indiens suggère que le processus de divergence entre ces deux types d'ADN date d'au moins 200000 ans, et serait donc bien antérieur à la période de domestication du néolithique. Ceci signifierait que Bos taurus et Bos indicus auraient été domestiqués à partir de deux populations sauvages distinctes, le foyer d'origine des zébus pouvant se situer dans l'actuel Pakistan.

Curieusement, tous les zébus africains semblent posséder en même temps le chromosome Y acrocentrique du zébu, et le type d'ADN mitochondrial des taurins, suggérant que l'introduction de gènes zébus en Afrique s'est faite essentiellement par la voie mâle [24]. Par ailleurs, après avoir identifié des allèles de microsatellites spécifiques du zébu indien (allèles absents chez les taurins européens), MacHugh et al. [25] constatent que la fréquence relative de ces allèles dans les populations de zébus africains décroît d'est en ouest, ce qui parait traduire le gradient d'introduction du zébu indien dans les populations taurines préexistantes. Tous ces résultats confirment que le peuplement bovin de l'Afrique, d'abord par les taurins à cornes longues, puis par les taurins à cornes courtes, et enfin par les zébus a été un processus d'une grande complexité.

Les populations bovines africaines ont fait l'objet de descriptions et d'inventaires variés, remarquablement synthétisés dans la monographie d'Epstein [9], ainsi que de nombreuses études d'ordre zootechnique. Par contre, le potentiel d'information représenté par les marqueurs biochimiques est loin d'avoir encore été exploité comme il l'a été pour les races européennes.

Les travaux effectués en Europe, à l'aide du polymorphisme des groupes sanguins et de certaines protéines du sang et du lait ont en effet permis de préciser les relations existant entre les races autrichiennes [19], ibériques [20, 13], italiennes [1], allemandes [14] et françaises [16]. Par ailleurs, une importante analyse synthétique avait été effectuée dès 1980 par Manwell et Baker. Ces auteurs ont montré que l'arbre phylogénétique de dix principaux groupes raciaux européens se superposait à la carte géographique, de telle manière que ces groupes se plaçaient dans des régions proches de leur centre présumé d'origine et de diversification, résultat qui donnait un crédit certain au classement phylogénétique obtenu [27].

La présente étude tente d'établir, à partir des fréquences alléliques de 16 systèmes polymorphes (groupes sanguins et protéines sériques), les relations génétiques existant entre dix populations bovines appartenant à cinq populations présentes en Afrique de l'Ouest, les populations taurines N'Dama (type à cornes longues), Baoulé, Lagunaire (type à cornes courtes) et les populations zébus Peuhl soudanaise et Azawak. Les analyses ont été effectuées de 1982 à 1991 au Centre de recherche sur les trypanosomoses animales (CRTA) de BoboDioulasso, au Burkina-Faso. Par ailleurs, la présence de 12 systèmes génétiques 
communs à cette étude de populations africaines et aux travaux de Grosclaude et al. [16] sur les races françaises a permis de réaliser une analyse d'ensemble de toutes ces populations, européennes et africaines, fondée sur ces 12 systèmes.

\section{MATÉRIELS ET MÉTHODES}

\subsection{Races et échantillonnages}

Les cinq «races» retenues dans cette étude sont les races taurines N'Dama, Baoulé et Lagunaire et les races zébus Peuhl soudanaise et Azawak.

Taurins N'Dama. Selon Epstein [9], le «berceau de race» de ce bétail est le plateau du Fouta-Djallon en Guinée, d'où il s'est répandu en Gambie, Sierra-Leone, Liberia et Mali occidental. Des troupeaux ont été constitués par la suite dans d'autres pays. Les animaux de cette étude proviennent de cinq origines : Guinée, Mali, Sénégal, Togo et Côte-d'Ivoire (figure 1). En Guinée, les animaux proviennent du Centre de recherches zootechniques de Boké. Au Mali, les animaux sont originaires du troupeau du ranch de MadinaDiassa, constitué entre 1975 et 1981 par des achats dans diverses régions du pays. Il a ainsi été créé une souche dite «Madina», de petit format et de couleur homogénéisée par l'élimination des animaux tachetés [32]. Au Sénégal, les animaux proviennent du Centre de recherches zootechniques de Kolda, relevant de l'Institut sénégalais de recherche agronomique (ISRA). Au Togo, les animaux proviennent du Centre de recherche et d'élevage d'Avetonou-Togo (CREAT), et descendent d'un type importé de Guinée, région d'origine du N'Dama, entre 1954 et 1974, par la Station d'élevage de Nassablé-Dapaong. En Côte-d'Ivoire, les animaux sont nés au Département d'élevage de l'Institut des savanes (Idessa) à Minankro, mais leurs ascendants proviennent principalement d'élevages villageois traditionnels du nord-ouest du pays, où les zébus n'ont pas été introduits.

Taurins Baoulé. Ce bétail à cornes courtes, adapté à la savane, est éponyme de l'ethnie qui l'élève dans le Centre de la Côte-d'Ivoire. Dans ce pays, les animaux retenus proviennent, comme les N'Dama, du département d'élevage de l'Institut des savanes, dont le troupeau a été constitué par des achats effectués au Nord-Est et au Centre du pays, dans les régions de Bouna-Dorogo et de Korhogo. Les taurins Baoulé du Burkina-Faso proviennent de Banankélédaga au Burkina-Faso.

Taurins Lagunaire. Ce type est une des composantes du groupe de taurins nains à cornes courtes, localisée au Bénin. Les animaux proviennent de Samiondji.

Zébus Peuhl soudanais. Il s'agit d'un type à bosse thoracique, à cornes en lyre chez le mâle, localisé au sud du Mali et au nord du Burkina-Faso. Les animaux proviennent de Banankélédaga au Burkina-Faso.

Zébus Azawak. Ce Zébu à cornes courtes et à bosse cervico-thoracique est élevé dans le bassin de l'Azawak au Mali oriental et au centre du Niger, ainsi que le long de la frontière nord-ouest du Nigeria. Les animaux proviennent de Filingué au Niger.

Les prises de sang ont été effectuées sur les mères et leur veau âgé de plus de 4 mois, ainsi que sur les pères de ces veaux lorsqu'ils étaient accessibles. Seules 


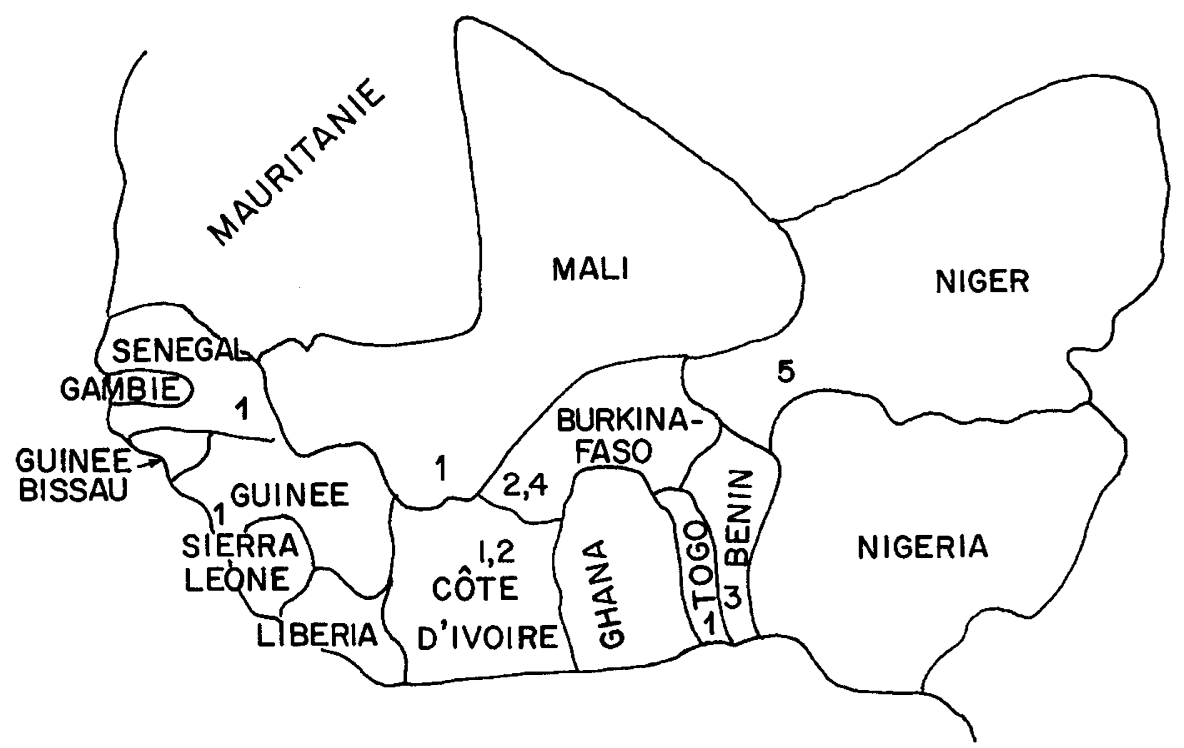

Figure 1. Localisation des différents sites de prélèvement. 1 : taurins $\mathrm{N}^{\prime}$ Dama; 2 : taurins Baoulé; 3 : taurins Lagunaires; 4 : zébus Peuhl soudanais; 5 : zébus Azawak.

les mères ont été retenues pour constituer les échantillons de populations, les résultats obtenus sur les veaux et les pères ne servant qu'à faciliter l'interprétation des génotypes maternels. L'effectif total des 2717 mères se répartit comme suit. 1) N'Dama : 1714 dont 714 du Mali, 185 du Togo, 374 de Côte-d'Ivoire, 192 de Guinée et 249 du Sénégal. 2) Baoulé : 740 dont 351 de Côte-d'ivoire et 389 du Burkina Faso. 3) Lagunaire : 50. 4) Zébu Peuhl : 138. 5) Zébu Azawak : 75 .

\subsection{Systèmes génétiques polymorphes}

L'analyse s'appuie sur le polymorphisme génétique des 11 systèmes de groupes sanguins érythrocytaires connus chez les bovins $(A, B, C, F, J, L, M, S$, $Z, R^{\prime}, T^{\prime}$ ) et de cinq systèmes protéiques : albumine sérique $(A l)$, transferrine sérique $(T f)$, hémoglobine $(H b)$, purine nucléoside phosphorylase $(N P)$ et phosphoglucomutase érythrocytaires ( $P G M 3)$. Le polymorphisme des deux systèmes de groupes sanguins complexes, $B$ et $C$, vraisemblablement codés par plusieurs gènes étroitement liés [15, 17], a été simplifié et ramené à ce qui peut être assimilé à de véritables séries alléliques, comme dans l'étude de Grosclaude et al. [16] sur les races françaises. Le système B a été réduit aux six allèles obtenus par regroupement des phénogroupes (haplotypes) comportant respectivement les facteurs antigéniques $G_{1}, I_{1}, K, P_{1}, T$ et $J^{\prime \prime}$, et à un septième allèle regroupant les phénogroupes résiduels. De même, les phénogroupes comportant $I_{1} K$ et $G_{1} K$ ont été classés avec le groupe allélique $(K), I_{1} T$ avec $(T)$ et $I_{1} G_{1}$ avec $\left(G_{1}\right)$. Le système C a été ramené à six allèles. Quatre d'entre eux, correspondent, comme dans Grosclaude et al. [16], aux ensembles de phénogroupes comportant respectivement les facteurs antigéniques $C_{1}, C_{2}, C_{1}^{\prime \prime}$ et $C_{2}^{\prime \prime}$. 
Les deux autres allèles, de dénomination provisoire, $C^{-}$et $C^{C_{1-}}$ correspondent à des phénogroupes qui, dans les limites de cette étude, sont spécifiques des populations africaines : $C_{-}$désigne des phénogroupes ne comportant aucun des quatre facteurs antigéniques précédents, et $C_{1-}$ des phénogroupes réagissant avec anti- $C_{1}$, mais pas avec anti- $C_{2}$.

L'ensemble allélique ainsi défini avec ces 16 systèmes génétiques compte au total 63 allèles, dont 47 sont statistiquement indépendants (tableau I). Parmi ces 16 systèmes, 12 étaient utilisés dans Grosclaude et al. [16] : les 11 systèmes de groupes sanguins et le système de la transferrine sérique.

\subsection{Techniques d'analyse}

Les réactifs de groupes sanguins, obtenus au CRTA de Bobo-Dioulasso ont été validés par référence à ceux produits par le laboratoire d'analyse des groupes sanguins de l'Inra, à Jouy-en-Josas, eux-mêmes validés à l'occasion des tests de comparaison internationaux [15]. Le polymorphisme de l'albumine, de l'hémoglobine, de la purine nucléoside phosphorylase et de la phosphoglucomutase a été mis en évidence par électrophorèse horizontale en gel d'acétate de cellulose, celui de la transferrine par électrophorèse en gel de polyacrylamide. La révélation des enzymes érythrocytaires $(N P, P G M 3)$ se fait par des méthodes chromogéniques, et celle des autres protéines $(A l, H b, T f)$ s'effectue par coloration non spécifique.

\subsection{Détermination des fréquences alléliques}

Les fréquences alléliques ont été calculées par comptage direct pour les systèmes génétiques où tous les allèles sont codominants $(T f, A l, H b, N P$, $P G M 3)$, par la méthode de la racine carrée pour les systèmes bi-alléliques comportant un allèle "négatif» $\left(A, J, L, M, Z, R^{\prime}\right.$ et $\left.T^{\prime}\right)$ et par la méthode itérative de Ceppellini et al. [6] pour les systèmes complexes $(B, C$ et $S)$.

\subsection{Analyse factorielle des correspondances (AFC)}

L'analyse factorielle des correspondances, ou AFC [22], est une méthode d'analyse multidimensionnelle, analogue à l'analyse en composantes principales et spécialement adaptée à l'étude des tableaux de contingence et de fréquences. Elle permet une représentation simultanée des observations et des variables, donc dans notre cas des races et des allèles. Elle a été effectuée avec la procédure Corresp du progiciel SAS ${ }^{\circledR}$ (version 6, SAS Institute 1989).

\subsection{Distances génétiques}

Deux distances génétiques ont été utilisées pour quantifier la dissemblance globale entre les races : la distance standard de Nei [29], ou $D_{S}$, et la distance de Cavalli-Sforza et Edwards [5], ou $D_{C}$. Ces deux distances sont les plus communément utilisées, car elles présentent des propriétés intéressantes : $D_{S}$, sous l'hypothèse que les populations en présence sont d'effectif efficace constant, permet d'estimer le temps de divergence entre deux populations. $D_{C}$, sous l'hypothèse que les populations en présence sont d'effectif efficace variable, permet d'estimer le temps de divergence entre deux populations. 


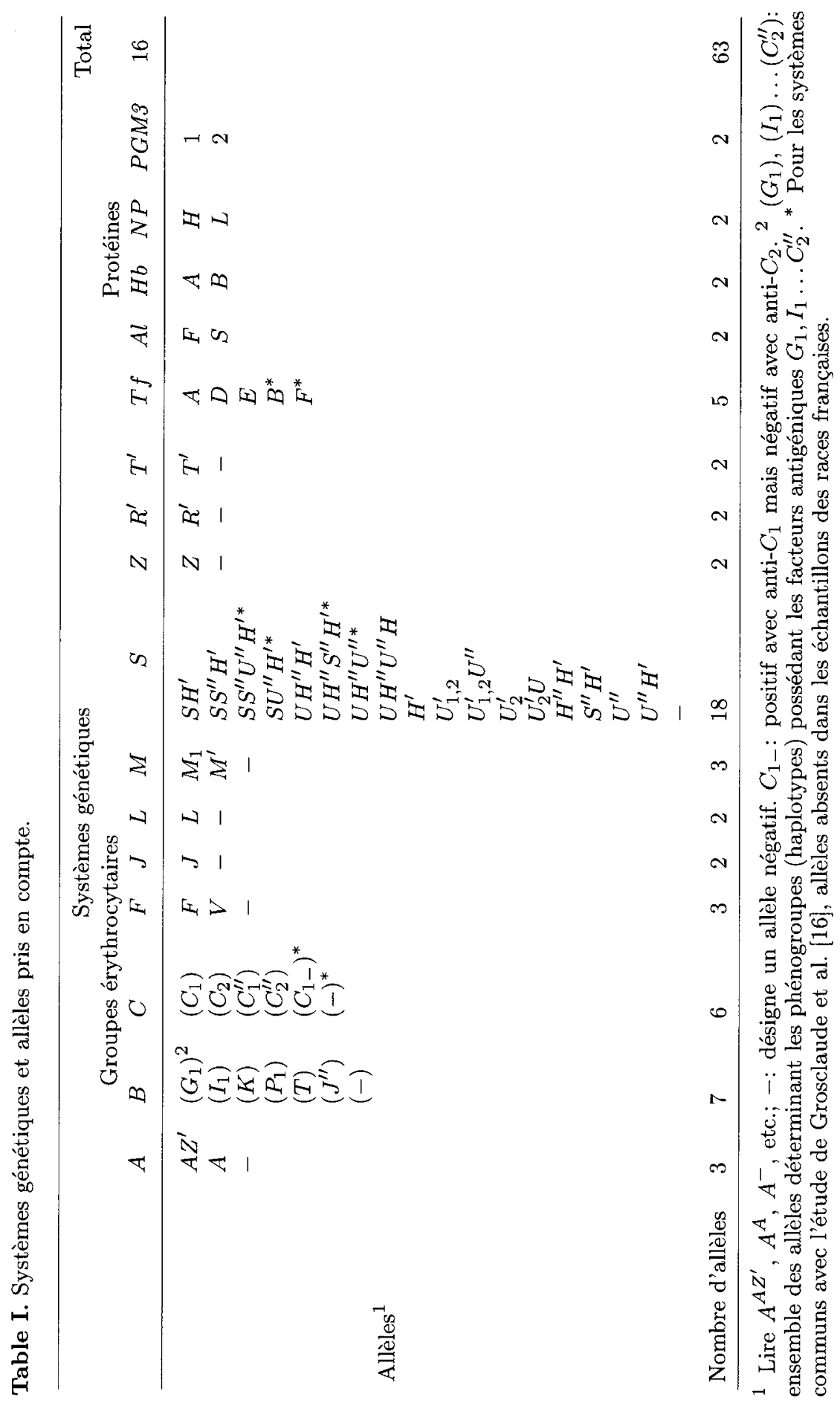




\subsection{Réalisation des phénogrammes}

La méthode d'agrégation dite «du plus proche voisin» (Neighbor Joining ou $N J$ ) développée par Saitou et Nei [33], et l'algorithme dit «du lien moyen» (UPGMA) [34] ont été utilisés pour construire des phénogrammes à partir des matrices de distances calculées. Les deux représentations arborées diffèrent par le mode de sélection du couple le plus proche. Avec l'algorithme UPGMA, c'est la plus petite distance absolue qui est choisie, alors que l'algorithme $N J$ utilise la plus petite distance après pondération par l'ensemble des distances. Ainsi, l'algorithme $N J$ n'impose aucune contrainte aux taux d'évolution entre les différentes populations. L'analyse de la robustesse de l'arbre a été réalisée par la méthode du bootstrap $[8,10]$. Cette méthode permet d'apprécier l'incertitude due à l'échantillonnage. Un tirage au hasard avec remise de $k$ caractères (ici les locus) parmi les $k$ constituant les données est effectué. Un arbre est construit après chaque ré-échantillonnage, ce processus étant réitéré 500 fois. Un arbre de consensus synthétise les 500 arbres ainsi obtenus, permettant de visualiser leurs concordances. La méthode de construction d'arbre de consensus utilisée par le programme est celle de l'arbre de consensus majoritaire [28]. Dans la comparaison de plusieurs arbres présentant des topologies différentes, le programme recherche les nœuds présents dans 50 à $100 \%$ des arbres. On considère qu'un nœud est d'autant plus stable que son pourcentage d'apparition est élevé. Dans la représentation donnée chaque nœud (regroupement) est accompagné de son pourcentage d'apparition (valeur de bootstrap). Dans des études analogues $[3,7,26]$, le seuil de stabilité du nœud est en général fixé à $75 \%$, les valeurs inférieures à $50 \%$ étant à considérer avec prudence. Les calculs ont été effectués à l'aide du logiciel Phylip [10].

\section{RÉSULTATS}

\subsection{Particularités alléliques des races africaines}

Le manque de familles complètes n'a pas permis d'établir les listes et d'estimer les fréquences des phénotypes (haplotypes) des sytèmes de groupes sanguins complexes des races africaines. La seule analyse un peu poussée a été celles du système $B$ de la race N'Dama, où 46 des phénogroupes existants ont pu être identifiés (non montré). Plus du quart de ces phénogroupes (13 sur 46) présente l'originalité d'associer des facteurs antigéniques qui le sont rarement dans les races françaises $\left(G_{1}, I_{1}, K, P_{1}, T\right)$. Le groupe $B G K$, en particulier, est de type $B G_{1} K$, alors qu'il est en général de type $B G_{2} K$ chez ces dernières. Par ailleurs, les phénogroupes du système $C$ des races françaises comportent toujours, sauf dans de très rares exceptions, l'un des quatres antigènes $C_{1}, C_{2}, C_{1}^{\prime \prime}$ ou $C_{2}^{\prime \prime}$. Chez les taurins comme chez les zébus africains, deux autres classes de phénogroupes sont observés : les phénogroupes de la première, dont la fréquence d'ensemble peut atteindre 0,23 , ne comportent aucun des quatre antigènes ci-dessus (allèle noté $C^{-}$); ceux de la seconde, dont la fréquence peut aller jusqu'à 0,68 , réagissent avec anti- $C_{1}$, mais pas avec anti- $C_{2}$ (allèle noté $C^{C_{1-}}$ ). Au système $\mathrm{S}$, deux «nouveaux» phénogroupes, peu fréquents, ont été observés dans les races africaines, $U H^{\prime \prime} S^{\prime \prime} H^{\prime}(f<0,03)$ et $U H^{\prime \prime} U^{\prime \prime}(f<0,01)$. Enfin, au système $\mathrm{F}$, l'allèle $F^{-}$(négatif avec anti- $F$ et 
anti- $V$ ) qui, sur plus de 650000 analyses effectuées dans les races françaises, n'a été soupçonné que dans une famille de race Charolaise, atteint une fréquence de l'ordre de 0,1 dans les races africaines, et même de 0,38 en race Lagunaire.

En ce qui concerne les protéines sanguines, l'allèle $\mathrm{B}$ de l'hémoglobine s'observe avec une fréquence de 0,35 à 0,40 chez les zébus, contre 0,08 au maximum chez les taurins africains. Par ailleurs, les allèles B et F de la transferrine, qui paraissent spécifiques des zébus, atteignent des fréquences de 0,03 à 0,05 pour le premier et de 0,25 à 0,32 pour le second.

\subsection{Construction de phénogrammes à partir de distances génétiques}

L'utilisation de deux distances génétiques $\left(D_{C}\right.$ et $\left.D_{S}\right)$ et deux méthodes d'agrégation (UPGMA et $N J$ ) permet d'obtenir quatre arbres. Les différences observées entre ces arbres sont dues à la méthode d'agrégation plus qu'à la distance génétique utilisée, car pour de faibles degrés de divergence, $D_{C}$ et $D_{S}$ sont fortement corrélées $(r=0,95)$. Dans les phénogrammes réalisés avec l'algorithme UPGMA sur les populations africaines (figure 2), trois ensembles se distinguent : le premier est constitué par les zébus (bootstrap $=85 \%$ ), le second par la seule race Lagunaire (bootstrap $=64 \%$ ) et le troisième par les taurins N'Dama et Baoulé. Dans ce dernier groupe, la population N'Dama de Guinée se distingue des autres (bootstrap = 85\%), mais, dans l'ensemble, les diverses populations N'Dama et Baoulé tendent à se regrouper par race et zone géographique. Les résultats obtenus avec l'algorithme $N J$ sont semblables. Toutefois, si les zébus paraissent encore plus nettement séparés (bootstrap= $99 \%$ avec la distance $D_{S}$ ) la différenciation des diverses populations taurines est beaucoup moins claire (non montré).

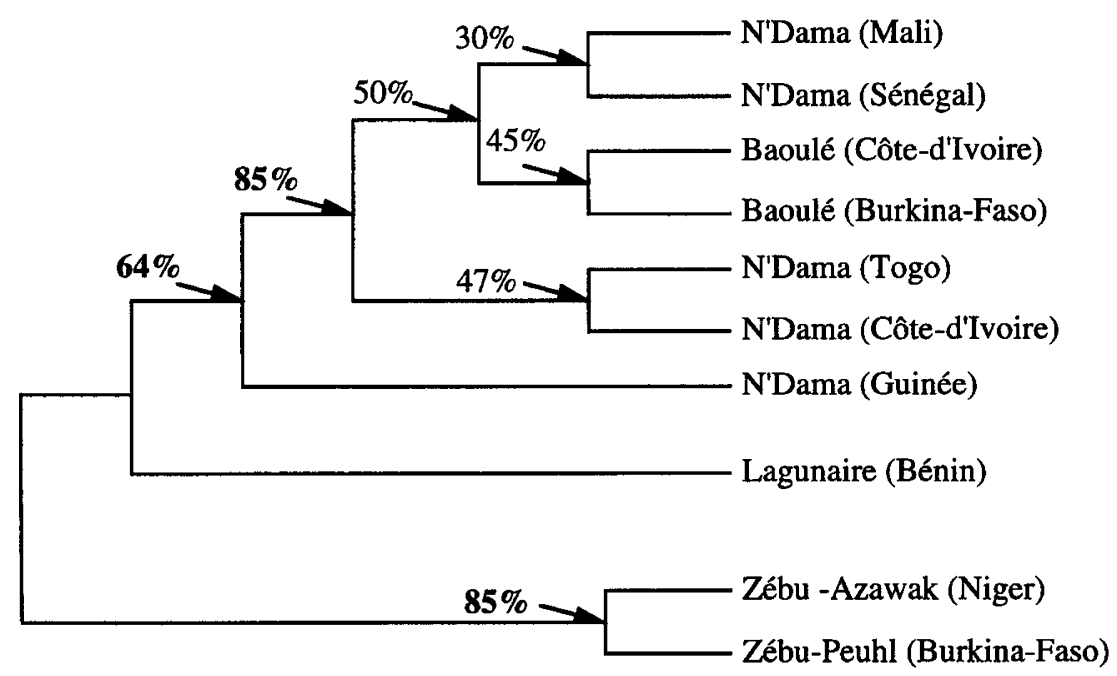

Figure 2. Populations africaines : arbre de consensus obtenu après 500 ré-échantillonnages des données (16 locus) en utilisant la matrice de distances de Cavalli-Sforza et Edwards et la méthode UPGMA. 
Dans l'analyse portant sur l'ensemble des races africaines et françaises l'opposition entre taurins et zébus se retrouve dans trois des quatre arbres (algorithme $N J$ avec $D_{C}$ et $D_{S}$, algorithme $U P G M A$ avec $D_{C}$ ) avec des valeurs de bootstrap variant de $60 \%$ à $82 \%$ (figure 3 ). Dans ces trois arbres, la séparation entre taurins africains et français est associée à des valeurs de bootstrap variant de $75 \%$ à $87 \%$ alors que, dans l'arbre calculé avec l'algorithme $U P G M A$ à partir de la distance $D_{S}$, les races africaines et françaises sont séparées avec une valeur de bootstrap plus faible (55\%). La position de la race Lagunaire varie suivant la méthode d'agrégation. Elle se rapproche des Baoulés avec l'algorithme $N J$ alors qu'elle s'en distingue avec l'algorithme UPGMA. Seuls les N'Dama du Togo et du Sénégal sont regroupés avec une forte valeur de bootstrap (75\% à $94 \%$ ). Les N'Dama du Mali, de la Côte-d'Ivoire et de la Guinée n'ont pas une position stable. Les Baoulés sont regroupés avec une valeur de bootstrap très variable suivant la méthode d'agrégation utilisée.

\subsection{Analyse factorielle des correspondances (AFC)}

La figure 4 représente les résultats de l'AFC concernant les seules races africaines. Trois groupes sont bien séparés : les zébus d'une part, les races Baoulé et N'Dama d'autre part et la race Lagunaire. Cinquante pour cent de la variation totale s'expliquent par le premier axe qui sépare les zébus des taurins. Trois allèles contribuent principalement à la construction de cet axe, les allèles $S$ de l'albumine, $F$ de la transferrine et $B$ de l'hémoglobine qui expliquent respectivement $21 \%, 13 \%$ et $7 \%$ de l'inertie de l'axe. Le deuxième axe explique $19 \%$ de la variation totale et distingue la race Lagunaire des autres taurins africains. La position isolée de cette race s'explique essentiellement par les trois allèles négatifs du système $\mathrm{B}$, du système $\mathrm{C}$ et du système $\mathrm{F}$ qui contribuent respectivement pour $10 \%, 11 \%$ et $11 \%$ à l'inertie expliquée par cet axe. Le retrait des trois systèmes les plus discriminants pour chacun des deux axes ne change pas fondamentalement la répartition des diverses races (non montré) ce qui traduit une bonne robustesse des résultats de l'analyse. La prise en compte des autres axes n'apporte aucun autre élément de structuration, notamment pour le groupe des populations N'Dama et Baoulé. Il en est de même lorsque les zébus et la race Lagunaire sont retirés de l'analyse (non montré).

La figure 5 représente les résultats de l'AFC appliquée à l'ensemble des races africaines et françaises. Le premier axe explique $28 \%$ de la variation totale et distingue clairement les races africaines des races françaises. L'opposition entre ces deux groupes de races s'explique essentiellement par trois allèles $\left(C^{C_{1-}}\right.$ du système $\mathrm{C}, F^{-}$du système $F$ et $A^{-}$du système $\mathrm{A}$ ) qui rendent compte respectivement de $22 \%, 12 \%$ et $8 \%$ de l'inertie de l'axe. $\grave{A}$ noter que le retrait de l'analyse des systèmes $\mathrm{C}, \mathrm{F}$ et $\mathrm{A}$ ne change pas fondamentalement la séparation des races selon le premier axe, ce qui est le signe d'une certaine robustesse des résultats. Le deuxième axe explique $17 \%$ de la variation et sépare les zébus des taurins. Les deux allèles qui influencent le plus cet axe sont l'allèle $\mathrm{F}$ de la transferrine, qui paraît spécifique des zébus, avec $35 \%$ de la variation expliquée par l'axe, et l'allèle $A^{A Z^{\prime}}$ du système $\mathrm{A}$ avec une contribution de $7 \%$. Le retrait de ces deux allèles n'apporte aucun autre élément de structuration. 


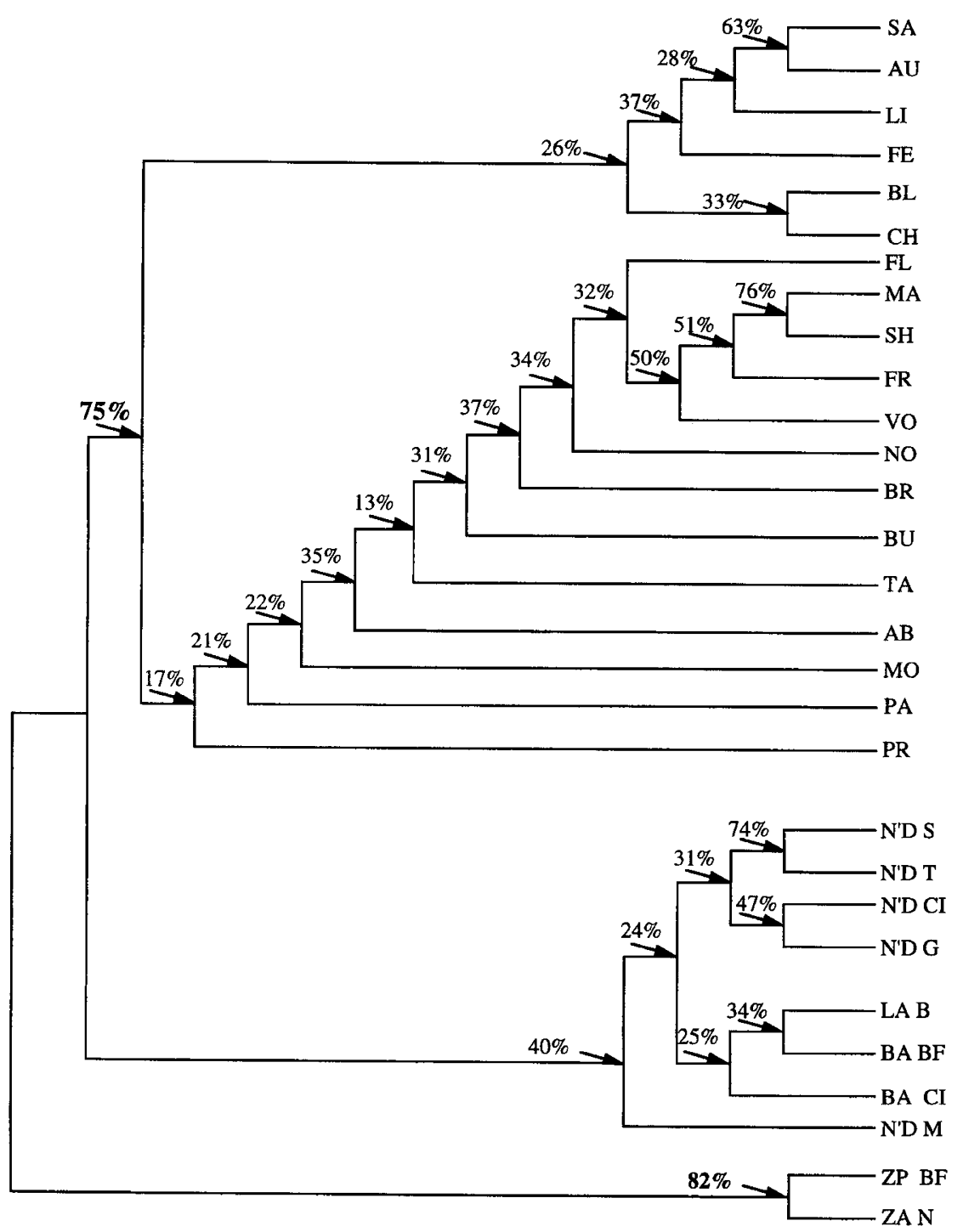

Figure 3. Populations françaises et africaines : arbre de consensus obtenu après 500 ré-échantillonnages des données (12 locus) en utilisant la matrice de distances de Nei et la méthode NJ. Désignations des races de haut en bas : $S A$, Salers; $A U$, Aubrac; $L I$, Limousine; $F E$, Ferrandaise; $B L$, Blonde d'Aquitaine; $C H$, Charolaise; $F L$, Flamande; $M A$, Maine-Anjou ; $S H$, Shorthorn; $F R$, Frisonne Pie-Noire ; $V O$, Vosgienne; No, Normande; $B R$, Bretonne Pie-Noire; $B U$, Brune; TA, Tarine; $A B$, Abondance; $M O$, Montbéliarde; $P A$, Parthenaise; $P R$, Pie-Rouge de l'Est; $N^{\prime} D S$, N'Dama du Sénégal; $N^{\prime} D T$, N'Dama du Togo; $N^{\prime} D C I$, N'Dama de Côte d'Ivoire; $N^{\prime} D G$, N'Dama de Guinée; $L A B$, Lagunaire du Bénin; $B A B F$, Baoulé du Burkina-Faso; $B A C I$, Baoulé de Côte d'Ivoire; $N^{\prime} D M$, N'Dama du Mali; $Z P B F$, Zébu Peuhl du Burkina-Faso; $Z A N$, Zébu Azawak du Niger. 


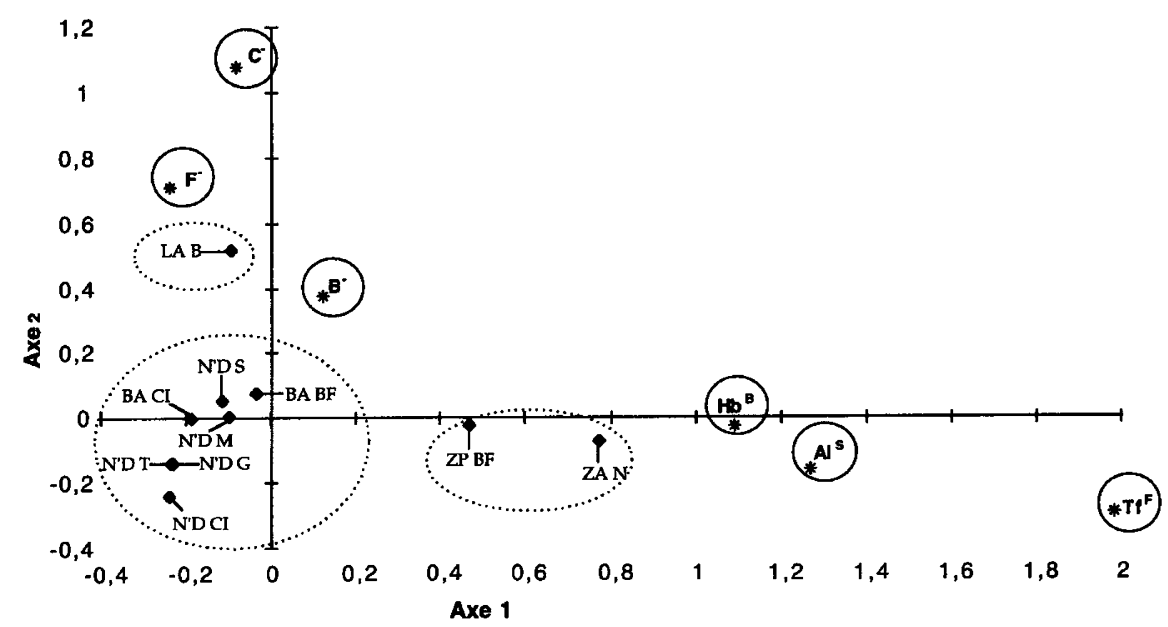

Figure 4. Analyse factorielle des correspondances : projection sur le plan des deux premiers axes, des races africaines et des six allèles les plus discriminants (encerclés). Pour la désignation des races voir légende de la figure 3.

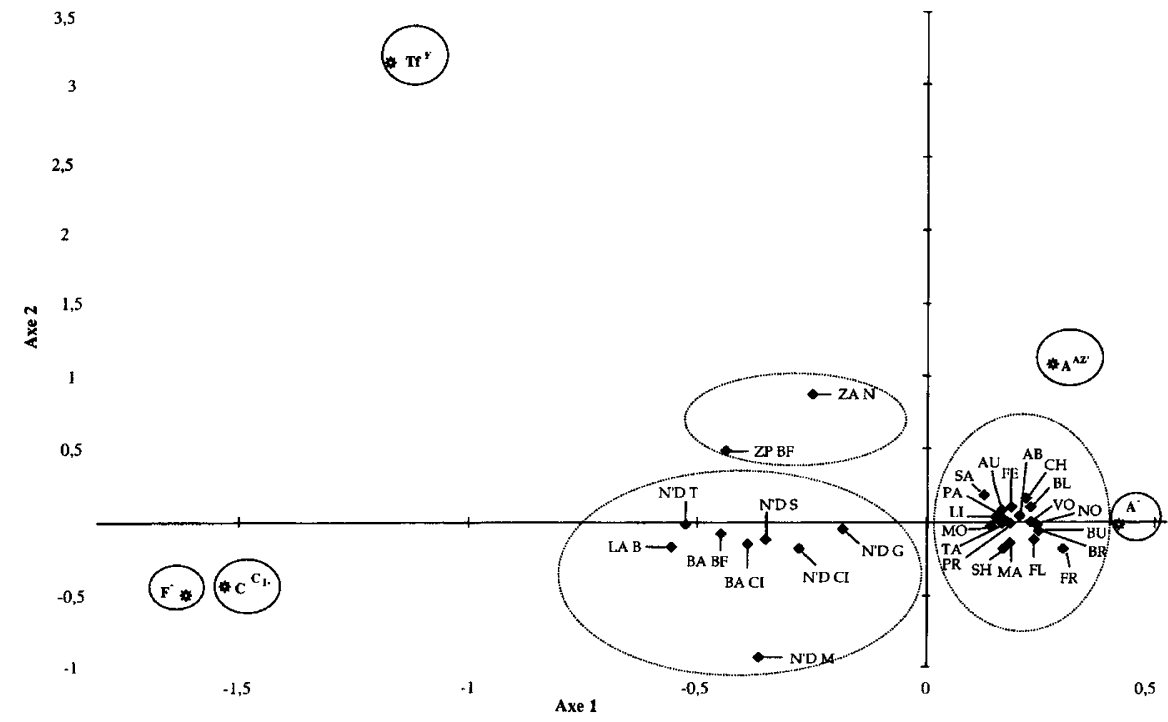

Figure 5. Analyse factorielle des correspondances : projection sur le plan des deux premiers axes des races africaines et françaises et des cinq allèles les plus discriminants (encerclés). Pour la désignation des races voir légende de la figure 3.

Le troisième et quatrième axe expliquent respectivement $11 \%$ et $9 \%$ de la variation totale. Ces deux axes (non montré) permettent d'une part de séparer la race Lagunaire et, d'autre part, pour les races françaises, de retrouver deux groupes (nord, centre et sud-ouest) parmi les quatre grands groupes mis en évidence par Grosclaude et al. [16]. 


\section{DISCUSSION}

Les microsatellites sont devenus les systèmes génétiques les plus couramment utilisés pour le marquage des génomes des animaux d'élevage. Ce type de marqueurs présente en effet plusieurs avantages, i.e. le nombre considérable de sites microsatellites dans le génome, leur polymorphisme souvent élevé et la facilité d'accès aux techniques d'analyse. Depuis le début des années quatre-vingt-dix, les microsatellites ont largement supplanté, chez les animaux domestiques, les marqueurs classiques précédemment utilisés dans les études de génétique des populations, essentiellement les groupes sanguins et les protéines du sang qui ne présentent pas le même ensemble d'avantages. Toutefois cette évolution ne remet pas en cause le bien-fondé de l'utilisation de ces marqueurs. La présente analyse des populations bovines d'Afrique de l'Ouest qui a été entreprise avant la mise en évidence de marqueurs microsatellites, est fondée sur 16 systèmes de marqueurs classiques. Elle présente la particularité d'utiliser 12 systèmes communs avec ceux du travail de Grosclaude et al. [16] sur les races françaises, ce qui a permis d'entreprendre une analyse d'ensemble portant sur 19 races européennes et sur des populations africaines appartenant à deux types zébus et trois types taurins. Les analyses effectuées dégagent clairement trois ensembles de populations : zébus, taurins africains et taurins européens.

Dans l'analyse factorielle des correspondances appliquée aux seules races africaines, les allèles contribuant le plus à la distinction entre zébus et taurins sont $A l^{S}$ et $H b^{B}$, nettement plus fréquents chez les zébus, ainsi que $T f^{F}$ qui n'est trouvé que chez ces derniers. Ces observations sont remarquablement cohérentes avec les données de la littérature puisque, comme l'établit la synthèse de Baker et Manwell [2], les zébus, qu'ils soient indiens ou africains, se caractérisent, pour les trois systèmes considérés et par rapport aux taurins, par une forte fréquence des allèles $A l^{S}$ et $H b^{B}$ et par la présence de $T f^{F}$. Dans l'analyse étendue aux races européennes les systèmes $A l$ et $H b$ ne sont pas utilisés, ce qui explique que, des trois allèles ci-dessus, seul $T f^{F}$ ressorte comme allèle discriminant les zébus des taurins. Le second allèle discriminant est $A^{A Z^{\prime}}$. Les locus $A$ et $H b$ étant étroitement liés [21], il est possible que l'allèle $A^{A Z^{\prime}}$ soit ici, en quelque sorte, un subsitut de l'allèle $H b^{B}$.

À la différence de l'analyse phénétique qui sépare d'abord les zébus des taurins, l'analyse factorielle des correspondances étendue à l'ensemble des populations distingue d'abord l'ensemble européen de l'ensemble africain. En fait, ce résultat est dû au poids des races européennes, qui représentent 19 des 29 populations étudiées. En opérant non pas sur les races, mais sur les trois groupes raciaux apparaissant dans la figure 5 (zébus, taurins africaines, taurins européens) le groupe des zébus est bien, à échelle identique, le plus excentré. Les deux méthodes donnent donc des résultats concordants. L'AFC présente l'avantage d'identifier les allèles les plus discriminants, en l'occurrence $C^{C_{1-}}, F^{-}$et $A^{-}$. L'allèle $C^{C_{1-}}$ ne semble pas avoir été observé chez les bovins d'Europe de l'Ouest et caractérise donc bien les populations africaines. L'allèle $F^{-}$a été découvert dès 1960 par Osterhoff [30] dans les races sud-africaines Drakensberger, Boran, Nguni, Bonsmara et Afrikander. Dans cette dernière, sa fréquence atteint 0,55 [31]. Cet allèle ne semblant exister dans certaines races européennes qu'à l'état sporadique [18], il peut être également considéré comme caractérisant les bovins africains. L'allèle $A^{-}$est ubiquitaire et sa corrélation 
avec le premier axe découle donc des différences de fréquence. Comme l'avait déjà noté Braend [4], il paraît particulièrement rare chez les taurins africains. On rappellera que cet allèle (en opposition avec $A^{A}$ ) contribue également à la discrimination entre races françaises [16].

En définitive, les conclusions de ce travail s'accordent bien avec les données de la littérature. Il est frappant de constater qu'il existe à la fois des particularités communes aux taurins et aux zébus de l'Afrique de l'Ouest et d'autres particularités communes aux taurins européens et aux taurins africains. Cet état de fait est forcément le résultat d'échanges entre populations de taurins et de zébus africains. Les travaux de MacHugh et al [25] illustrent en effet la complexité de la situation, puisque les zébus africains possèdent le gonosome Y de Bos indicus, mais un ADN mitochondrial de Bos taurus et un génome autosomal mixte, où la proportion de gènes présumés taurins ou zébus varie selon les races.

Outre l'échantillon de type Lagunaire, le groupe des populations taurines africaines comportait cinq populations de type N'Dama et deux de type Baoulé, issus, au sein de chaque type, de pays différents. Les analyses effectuées n'aboutissent pas à un regroupement complet de ces populations par race, comme on aurait pu s'y attendre compte tenu du fait que N'Dama et Baoulé se rattachent à des vagues de peuplement bovin de l'Afrique différentes, les taurins à cornes longues pour la première, et les taurins à cornes courtes pour la seconde. Ce résultat est sans doute la conséquence de phénomènes de métissage ou d'absorption entre taurins, au bénéfice, pour la période récente, du N'Dama, plus productif [9], et peut être aussi d'un degré d'introgression variable de gènes zébus dans les populations taurines, qui semble attesté par les résultats de MacHugh et al. [25]. Les efforts faits au cours des dernières décennies pour reconstituer des troupeaux d'animaux de phénotype standard n'ont certainement pas pu éliminer tous les apports génétiques exogènes. On notera que les analyses effectuées sur les races françaises ([16] et figure $3 \mathrm{du}$ présent travail) ont dégagé un phénomène inattendu similaire, la proximité génétique des races Aubrac et Salers, que les auteurs de référence rattachent pourtant à des types morphologiques différents. Les aires d'extension de ces deux races sont contiguës et légèrement chevauchantes [35].

Le plateau de Fouta Djallon, en Guinée, est considéré comme le foyer d'origine du N'Dama, «la plus typique des races sans bosse à longues cornes rencontrées en Afrique de l'Ouest et la plus proche, morphologiquement, du Bos taurus primigenius de Rütimeyer» [9]. Or l'échantillon de N'Dama de Guinée inclus dans ce travail est le seul des échantillons d'Afrique où n'a été détecté aucun des cinq allèles paraissant les plus spécifiques des populations bovines africaines $\left(C^{-}, C^{C_{1-}}, F^{-}\right)$, ou tendant à y être plus fréquents $\left(A l^{S}, H b^{B}\right)$. Le N'Dama de Guinée apparaît donc comme la plus taurine des populations africaines étudiées. Cette particularité peut expliquer pourquoi, dans l'analyse phénétique entre populations africaines (figure 2), il se distingue significativement des autres populations N'Dama et Baoulé. Elle justifie l'idée [25] que les N'Dama de Guinée sont des taurins très peu touchés par les introgressions de gènes zébus.

La race Lagunaire, qui tend à se dégager des autres populations taurines (figures 2 et 4), s'en distingue notamment par des allèles «africains » $C^{-}$et $F^{-}$. La fréquence de $F^{-}$dans cette race est à rapprocher de celle observée 
dans la race Afrikander (0,55). Selon Frisch et al. [12] le type Sanga du sud de l'Afrique, type bovin à bosse cervico-thoracique auquel se rattache la race Afrikander, est à classer comme Bos taurus. Ceci peut conduire à supposer que l'allèle $F^{-}$est originaire du pool génique taurin de l'Afrique. De manière générale, les résultats du présent travail incitent à tenter de rechercher l'origine de certains allèles fréquents dans les populations africaines.

Le peuplement bovin de l'Afrique présente une complexité sans égale. Les travaux récents sur le polymorphisme de l'ADN mitochondrial et des microsatellites ont abouti à des résultats qu'il reste, sur certains points, à rendre cohérents avec le corps des données antérieures. Des inventaires complémentaires sont indispensables à partir d'échantillons suffisamment importants et représentatifs, en faisant appel à une panoplie de marqueurs dont les limites de fiabilité sont correctement appréciées.

\section{REMERCIEMENTS}

La réalisation de ce travail a bénéficié de la participation et de la collaboration de nombreuses personnes. Nous remercions les éleveurs, les responsables des services zootechniques, des stations ou ranchs d'élevage et leurs agents qui ont autorisé et contribué à la collecte des échantillons. Nous remercions également S. Sylla et A. Zoungrana pour leur collaboration technique (groupes sanguins, variants électrophorétiques). Nous témoignons notre gratitude à M.T. Alaux, D. François, G. Houlier, L. Méténier et G. Ruffet du Laboratoire des groupes sanguins, M. Boitard, M. Wimitzky du Laboratoire de génétique factorielle, et $\mathrm{B}$. Bonaiti de la station de génétique quantitative et appliquée, pour leurs conseils et l'intérêt qu'ils ont porté à cette étude.

Ce travail a été réalisé avec le support financier de l'Institut d'élevage et de médecine vétérinaire des pays tropicaux (IEMVT), Maisons-Alfort, France, département du Centre de coopération internationale en recherche agronomique pour le développement (Cirad) et de la Deutsche Gesellschaft für Technische Zusammenarbeit (GTZ), P.N 77.2275, Eschborn, Allemagne.

\section{RÉFÉRENCES}

[1] Astolfi P., Pagnacco G., Guglielmino-Matessi C.R., Phylogenetic analysis of native Italian cattle breeds, Z. Tierz. ZüchtBiol. 100 (1983) 87-100.

[2] Baker C.M.A., Manwell C., Chemical classification of cattle. 1. Breed groups, Anim. Blood Groups Biochem. Genet. 11 (1980) 127-150.

[3] Bowcock A.M., Ruiz-Linares A., Tomfohrde J., Minch E., Kidd J.R., CavalliSforza L.L., High resolution of human evolutionary trees with polymorphic microsatellites, Nature 368 (1994) 455-457.

[4] Braend M., Blood groups of Nigerian cattle. Comparative aspects, Anim. Blood Groups Biochem. Genet. 10 (1979) 49-56.

[5] Cavalli-Sforza L.L., Edwards A.W.F., Phylogenetic analysis: models and estimation procedures, Evolution 21 (1967) 550-570.

[6] Ceppellini R., Siniscalco M., Smith C.A.B., The estimation of gene frequencies in a random mating population, Ann. Hum. Genet. 20 (1956) 97-115.

[7] Deka R., Jin L., Shriver M.D., Yu L.M., DeCroo S., Hundrieser J., Bunker C.H., Ferrell R., Chakraborty R., Population genetics of dinucleotide (dC-dA)n (dG-dT)n polymorphism in world populations, Am. J. Hum. Genet. 56 (1995) 461474 . 
[8] Efron B., Bootstrap methods: an other look at the jacknife, Ann. Statist. 7 (1979) 1-26.

[9] Epstein H., The Origin of Domestic Animals of Africa, Vol. 1, Africana publishing corporation, New York, 1971.

[10] Felsenstein J., Confidence limits on phylogenies: an approach using the bootstrap, Evolution 39 (1985) 783-791.

[11] Felsenstein J., PHYLIP - Phylogeny Inference Package, Version 3.5 Edition, Departement of Genetics, Washington University, Seattle, 1993.

[12] Frisch I.E., Drinkwater R., Harrison B., Johnson S., Classification of the southern African Sanga and East African shorthorned Zebu, Anim. Genet. 28 (1997) $77-83$.

[13] Gonzalez P., Tuñon M.J., Vallejo M., Genetic relationships between spanish native breeds of cattle, Anim. Genet. 18 (1987) 249-256.

[14] Graml R., Schmid D.O., Erhardt L., Buchberger J., Ohmayer G., Pirchner F., Verwandtschaft des Murnau-Werdenfelser Rindes zu anderen Rassen, Bayer. Landwirtsch. Jahrb. 63 (1986) 273-281.

[15] Grosclaude F., Guérin G., Houlier G., The genetic map of the B system of cattle blood groups as observed in French breeds, Anim. Blood Groups Biochem. Genet. 10 (1979) 199-218.

[16] Grosclaude F., Aupetit R., Lefebvre J., Mériaux J.C., Essai d'analyse des relations génétiques entre les raçes bovines françaises à l'aide du polymorphisme biochimique, Genet. Sel. Evol. 22 (1990) 317-338.

[17] Guérin G, Grosclaude F, Houlier G, The C system of cattle blood groups. II. Partial genetic map of the system, Anim. Blood. Groups. Biochem. Genet. 12 (1981) $15-21$.

[18] Hall J.G., Ross D.S., Evidence for the presence of an additional allele in the F system of British Friesan cattle blood, Anim. Blood. Groups. Biochem. Genet. (1982) $229-240$.

[19] Kidd K.K., Pirchner F., Genetic relationships of Austrian cattle breeds, Anim. Blood. Groups. Biochem. Genet. 2 (1971) 145-158.

[20] Kidd K.K, Stone W.H., Crimella C., Carenzi C., Casati M., Rognoni G., Immunogenetic and population genetic analyses of Iberian cattle, Anim. Blood. Groups. Biochem. Genet. 11 (1982) 21-38.

[21] Larsen B., Blood group and polymorphic proteins in cattle and swine, Ann. Génét. Sél. Anim. 3 (1971) 59-70.

[22] Lebart L., Morineau A., Tabart N., Techniques de la description statistique, Dunod, Paris, 1977, p 351.

[23] Lhoste P., Cattle genetic resources of west Africa, in: Hickman C.G. (Ed.), Cattle Genetic Resources, World Animal Science B: Disciplinary Approach, Elsevier, Amsterdam, 1991, pp. 73-88.

[24] Loftus R.T., MacHugh D.E., Bradley D.G., Sharp P.M., Cunningham P., Evidence for two independent domestications of cattle, Proc. Natl. Acad. Sci.USA 91 (1994) 2757-2761.

[25] MacHugh D.E., Shriver M.D., Loftus R.T., Cunningham P., Bradley D.G., Microsatellite DNA variation and the evolution, domestication and phylogeography of taurine and zebu cattle (Bos taurus and Bos indicus), Genetics 146 (1997) 1071-1086.

[26] MacHugh D.E., Loftus R.T., Bradley D.G., Sharp P.M., Cunningham P., Microsatellite DNA variation within and among European cattle Breeds, Proc. R. Soc. Lond B. 256 (1994) 25-31.

[27] Manwell C., Baker C.M.A., Chemical classification of cattle. 2. Phylogenetic tree and specific status of the Zebu, Anim. Blood Groups Biochem. Genet. 11 (1980) 151-162. 
[28] Margush T., McMorris F.R., Consensus N-trees, Bull. Math. Biol. 43 (1981) 239-244.

[29] Nei M., Genetic distance between populations, Am. Nat. 106 (1972) 283-292.

[30] Osterhorff D.R., Report of the South-African Blood Group Laboratory, Proceedings of a meeting of cattle blood group workers, Edinburgh, 23-26 August 1960, ABRO, Edinburgh, 1960, pp. 66-93.

[31] Osterhoff D.R., Politzer N., $F^{f}-$ A new allele in the bovine FV blood group system, Proceedings of the XI th European Conference on Animal Blood Groups and Biochemical Polymorphism (Warsaw, 1968), 1970, pp. 135-142.

[32] Planchenault D., Tall S.H., Traore M.T., Amélioration génétique des bovins N'Damas. Etudes en milieu extensif au Mali. 1. Caractéristiques du bétail N'Dama au ranch de Madina-Diassa, Rev. Elev. Méd. Vét. Pays. Trop. 37. (1984) 488-495.

[33] Saitou N., Nei M., The neighbour-joining method: a new method for reconstructing phylogenetic trees, Mol. Biol. Evol. 4 (1987) 406-425.

[34] Sneath P.H.A., Sokal R.R., Numerical Taxonomy, W.H. Freeman, San Francisco, 1973.

[35] Vissac B., Etude génétique de la race d'Aubrac, in: l'Aubrac, CNRS, Paris, I, 1970, pp. 29-102. 